\title{
TMHF: Transmit Max Hop First forwarding Strategy to optimize the Performance of Epidemic Routing Protocol
}

\author{
Qaisar Ayub, Sulma Rashid, M. Soperi Mohd Zahid \\ Faculty of Computer Science \& Information System, \\ Department of Computer System \& Communication \\ Universiti Teknologi Malaysia (UTM) Skudai - Johor, 81310, Malaysia.
}

\begin{abstract}
Delay Tolerant Networks vary from conventional networks in that they have reserve constraints, unbalanced mixture traffic, data redundancy, network dynamics, and energy balance. Moreover due to node mobility DTN suffers from unpredictable connectivity and limited transmission duration. A mobile node in DTN routing may not be able to transmit all messages from its forwarding queue; therefore the order in which messages are transmitted becomes very important.

In this paper, we propose forwarding queuing mechanisms named Transmit Max Hop Count first (TMHF) in which when the connection opportunity arises, the node will transmit the message with max hop count.
\end{abstract}

\section{General Terms}

Algorithm, Routing, Epidemic router.

\section{Keywords}

Store and forward networks; Forwarding strategies, routing,

DTN, Queue modes, maximum hop forward

\section{INTRODUCTION}

In conventional routing schemes TCP/IP it is necessary to launch end-to-end path from source to destination previous to the transmission of data. Hence most of wireless applications such as sensor networks for ecological monitoring [16], ocean sensor networks [18], [17], biological sensor networks [7], [11] and vehicular networks [19], [20] due to highly unstable path which may change or break while being discovered.

Delay tolerant networks (DTNs) permit the transmission of data by means of intermittently connected mobile nodes. DTN as [9],[4],[10],[6] put forward as work by via store-carry-forward paradigm, where every node acts as a hop in the network and store the message in buffer, carries the message while moving and forward when it encounter with another node.

Foundation on the scheme of message forwarding, routing plan for DTN can be separated in to two main categories, single copy and multi copy [12]. Here single copy routing schemes only one copy of message exists in the network, which is forwarded along single path [5] for example first contact [5], direct delivery [5]. While in multi copy schemes more then one copy of same message are forwarded to multiple paths for example Epidemic router[8], spray \& wait[14] ,prophet[3], MaxProp [20] ,probabilistic forwarding[3]. Multi copy routing cases according to [13] have high influence on message delivery probability and robustness at the price of extra bandwidth, energy and memory usage such as epidemic, spray \& wait.

Extensive delays, restricted bandwidth and limited duration of contact time, a node in epidemic router [8] can not be able to exchange all messages from its forwarding queue. Therefore the sequence in which the messages are transmitted becomes very vital. Additional by apply efficient buffer management policies and forwarding strategies we can optimized the performance of routing protocols .A small number of recently work [1], [21],[2], [24], [23],[22] and [15] have proposed few forwarding and buffer management strategies.

In this paper we have propose the forwarding strategy TMHF with existing forwarding queue FIFO to obtain the quality of service optimization in the performance of DTN routing in terms of delivery probability, overhead ratio and Hop count average.

The remaining paper is prearranged as follows .Section 2 elaborates existing forwarding strategies. Section 3 is about protocol under observation, Section 4 is performance metrics, Section 5, 6 are regarding approach and Algorithms .Simulation and results simulates in section 7 by a conclusion at section 8 .

\section{EXISTING FORWARDING STRATEGIES}

\subsection{First in First out (FIFO)}

In FIFO queue mode all messages are arranged according to arrival time and the message which has oldest arrival time will be transmitted first.

\subsection{Random Queue Mode (RND)}

The message is randomly selected for the transmission.

\subsection{GRTR}

"Assume A, B are nodes that meet while the destination is D, $\mathrm{P}_{(\mathrm{X}, \mathrm{Y})}$ denote the delivery predictability that a node $\mathrm{X}$ has for Destination Y. GRTR forward the message to node only if $\mathrm{P}_{\text {(B- }}$ D) $>P_{(A-D)} "[1]$.

\subsection{GRTRSort}

"GRTRSort looks at difference $\mathrm{P}_{(\mathrm{B}-\mathrm{D})}-\mathrm{P}_{(\mathrm{A}-\mathrm{D})}$ values for each message between the nodes and forward the message only if $\mathrm{P}_{(\mathrm{B}-}$ D) $>P_{(A-D)} \cdot "[1]$ 


\subsection{GRTRMax}

"Select messages in descending order of $\mathrm{P}_{(\mathrm{B}-\mathrm{D})}$ forward the message only if $\mathrm{P}_{(\mathrm{B}-\mathrm{D})}>\mathrm{P}_{(\mathrm{A}-\mathrm{D})}$." [1]

\subsection{TSMF}

In TSMF the forwarding queue the message with small size is placed on top of queue [2].

\section{PROTOCOL UNDER EVALUATION}

\subsection{Epidemic router}

In epidemic routing[8] application messages are swamped to the relay nodes called carriers , when carrier nodes while moving comes in contact with another related proton of network, it diffuse the message to further island of nodes. This cyclic forwarding increases the probability of message delivery to its destination.

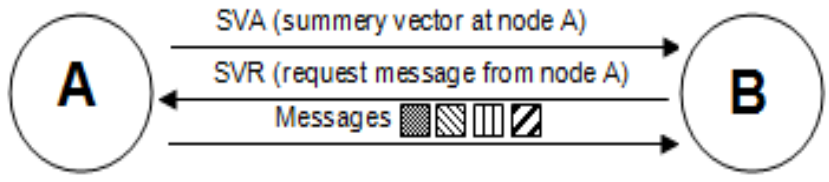

Fig 1 Epidemic Router

Fig 01 illustrates the flow of epidemic router, when Node A, B comes in the transmission range, node A exchanges its summery vector with node B. A summery vector is maintained locally on each node and it represents the buffered messages. After exchange of summery vector Node B by performing a logical AND operation between the negations of its summery vector and filter out messages not buffered at B (SVR). Node B transmits a request summery vector SVR to A The process prolong as when node $\mathrm{B}$ comes in contact with other node as a result increase the delivery probability on the cost of buffer space and transmission time.

\section{PERFORMANCE METRICS}

\subsection{Delivery probability}

It is the ratio of message received over message send. High probability means that more messages are delivered to the destination.

\subsection{Overhead-ratio}

It is the negation of number of messages relayed to number of message delivered. Low value of overhead means less processing required delivering the relayed messages. Objective of algorithm is to minimize the value of overhead.

\subsection{Hop count average}

It is the Mean hops which a message takes to reach its destination.

Table 1. Snapshot of node messages

\begin{tabular}{|c|c|c|c|c|}
\hline Node & Message_id & HC & Arrival Time & Destination \\
\hline \multirow{2}{*}{ A } & M4 & 10 & 15 & B \\
\cline { 2 - 5 } & M2 & 8 & 20 & B \\
\hline
\end{tabular}

\begin{tabular}{|c|c|c|c|c|}
\hline \multirow{2}{*}{} & M1 & 3 & 4 & C \\
\cline { 2 - 5 } & M51 & 5 & 3 & D \\
\hline \multirow{3}{*}{ B } & M3 & 9 & 45 & A \\
\cline { 2 - 5 } & M5 & 3 & 32 & D \\
\cline { 2 - 5 } & M43 & 5 & 34 & C \\
\hline \multirow{3}{*}{ C } & M18 & 4 & 12 & D \\
\cline { 2 - 5 } & M76 & 7 & 43 & C \\
\cline { 2 - 5 } & M32 & 8 & 76 & D \\
\hline
\end{tabular}

\section{APPROACH}

Assume A, B are two intermittently connected mobile node, $\mathrm{M}$ $\mathrm{A}$ represents the message buffered at node $\mathrm{A}$ where $\mathrm{MA}=\{$ M4,M2,M1,M51 $\}$ and Arrival Time ATA $=\{15,20,4,3\}$, MB represents the messages buffered at node $\mathrm{B}$ where $\mathrm{MB}=$ $\{\mathrm{M} 3, \mathrm{M} 5, \mathrm{M} 43\}$ and Arrival time of messages at $\mathrm{B}$ is ATB= $\{45,32,32\}$ while MC represents message buffered at node C. Further destination of buffered messages MA (M2, M4) is B, MA (M1, M51) is (C, D). Similarly the destination of messages stored at MB (M3, M5, and M43) is (A, D, C). We further assume each message transmission time is $1 \mathrm{~s}$ while total DTN transmission time is $2 \mathrm{~s}$.

\section{CASE 01: Forwarding sequence with FIFO queue mode}

Fig 02 depicts the exchange of messages using FIFO under epidemic routing protocol. Fig 02(a) represents the array of messages at node $\mathrm{A}$ before the transmission.

From Fig 02(b) we can examine that with available DTN transmission time message M3 and M1will be forwarded to node B while the destination of messages (M3, M1) is (C, D).
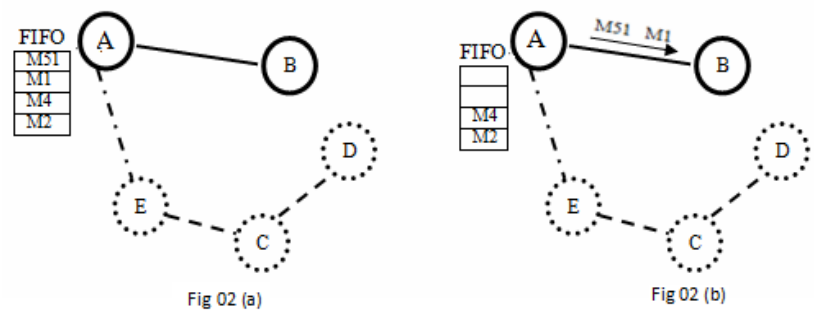

Fig 02 Message Transmission A-B using FIFO

Fig 03(a) demonstrates the string of messages at node $B$ by FIFO forwarding strategy. We can spot in Fig 03(b) that the M5 and M43 will be forwarded to node A; moreover the destination of (M5, M43) is (D, C). 

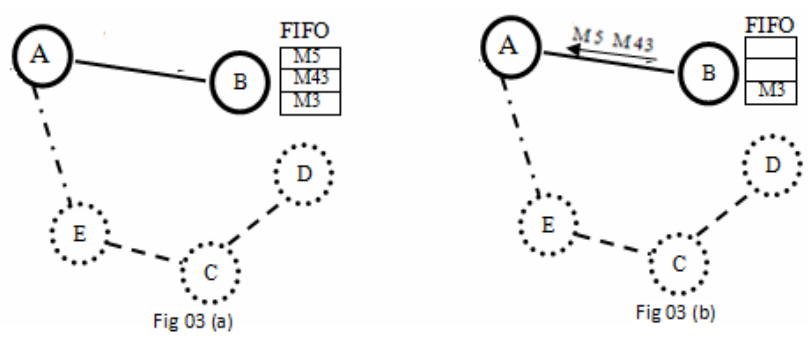

Fig 03 Message Transmission B-A using FIFO

Table 2. FIFO after Transmission

\begin{tabular}{|c|c|c|}
\hline Node & Messages & Destination \\
\hline A --- B & M51,M1 & C,D \\
\hline B----A & M5,M43 & D,C \\
\hline
\end{tabular}

Table 2 illustrates the result after the transmission involving A and B. After the messages were flooded from A to B (M51, M1) and from B to A (M5, M43), none of them found the destination.

\section{CASE 02: forwarding sequence with TMHF queue mode}

Fig 04(a) depicts the organization of messages at node A by TMHF previous to the transmission. Accordingly message M4; M2 will be forward to node B while the destination of (M4, M2) is $\mathrm{B}$ which results in raise of delivery ratio Fig-4.0 (b).
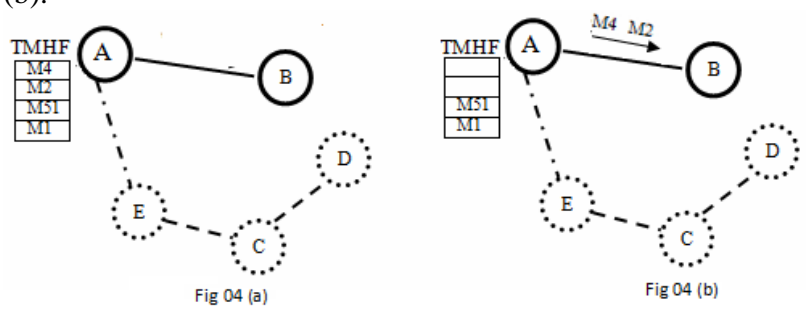

Fig 4 Message transmission A-B using TMHF
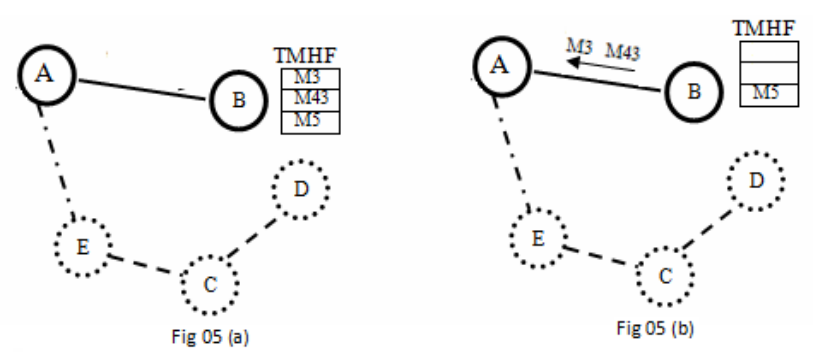

Fig 05 Message Transmission B-A TMHF

Fig 05(a) symbolizes the sequence of messages at node B using TMHF. From fig 05(b) we can see that M3, M43 will be transmitted to node A. While the destination of messages \{(M3, M43) $\}$ are (A, D).
Table 3. TMHF after Transmission

\begin{tabular}{|l|c|c|}
\hline Node & Messages & Destination \\
\hline A --- B & M4,M2 & B \\
\hline B----A & M3,M43 & A,D \\
\hline
\end{tabular}

Table 3 represents the result after the transmission of messages by THMF. We can distinguish that M4, M2 were forwarded from $\mathrm{A}$ to $\mathrm{B}$. The destination of both messages (TABLE 01) is B. Correspondingly when message replicate from B to A, M3 was delivered.

\section{Algorithm}

Step 01

Get messages from the router in List. Step 02

Order the messages by placing max hop to top of List.

OrderMessage (List)

Step 03

Transmit messages.

\section{SIMULATION AND RESULTS}

\subsection{By Varying Time Intervals}

Table 4. Simulation parameters w.r.t Time

\begin{tabular}{|l|l|}
\hline Number of Nodes & 120 \\
\hline Movement model 1 & Random Waypoint \\
\hline Router & Epidemic \\
\hline Number of groups & 03 \\
\hline Buffer size & $2 \mathrm{MB}$ \\
\hline Transmission range & $10 \mathrm{M}$ \\
\hline Transmission speed & $250 \mathrm{~K}$ \\
\hline Message creation & $30-40$ (seconds) \\
\hline Simulations times & $80 \mathrm{k}-560 \mathrm{k}$ \\
\hline Area & $4500 \mathrm{~m} \times 3400 \mathrm{~m}$ \\
\hline TTL & Infinity \\
\hline
\end{tabular}

In the following section we study the performance of TMHF under epidemic routing protocols, with exiting FIFO.

All the experiments were evaluated by ONE Simulator. The ONE Simulator is a discrete event simulator written in Java. The major aim of simulator is to relate DTN (store-carry-forward) of message for long time, where the probability of disconnections is high.

The Fig 06 depicts the comparison of FIFO and TMHF forwarding policy with respect to delivery probability. We can observe that by increasing simulation time the delivery probability of TMHF perform well then FIFO. 


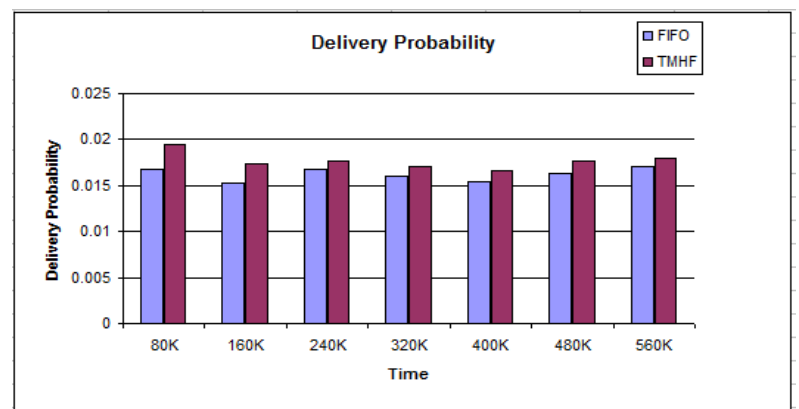

Fig 06 Delivery Probability w.r.t Time

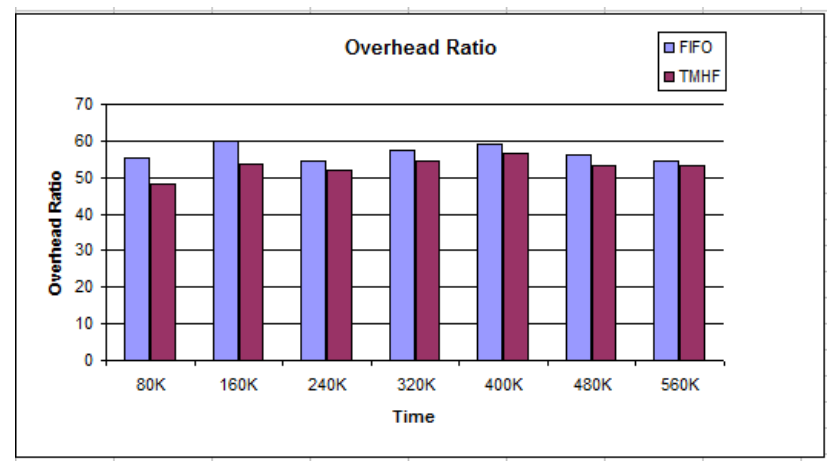

Fig 07 Overhead ratio w.r.t Time

Fig 07 simulates the effect of overhead ratio of FIFO and TMHF by varying the time interval from $80 \mathrm{~K}-160 \mathrm{~K}$ under epidemic router. Overhead metric can be used to calculate the consumption of resources, operational instructions during the message transmission.

The objective of algorithm must be to minimize the Overhead. We can see that overhead of proposed TMHF is lower then the FIFO.

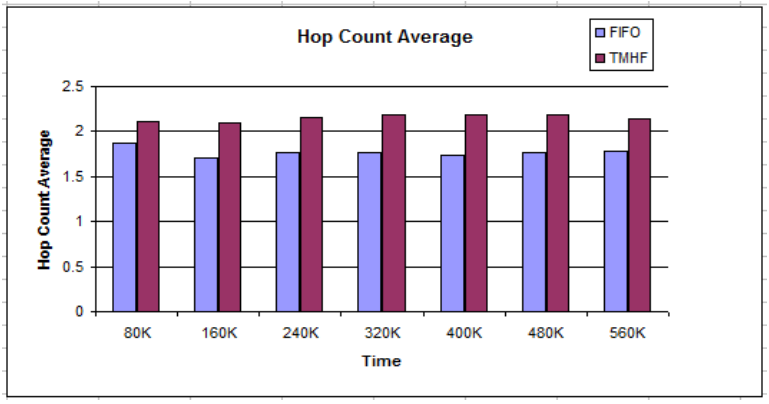

Fig 08 Hop count average w.r.t Time

In fig 08 we find the effect of hop count for FIFO and proposed TMHF under epidemic router. It can see clearly that at various time intervals the hop-count average of TMHF is higher then FIFO. High value of hop count reflects the fact that message is moving around the network work for long time. This reason of keeping message alive is that transmitting the message with greater hop already consumes the network resource; by forwarding such message (TMHF) may have the higher probability of it delivery as proved by fig.

\subsection{By Varying Area (Sparse)}

Table 5. Simulation parameters w.r.t Area

\begin{tabular}{|l|l|}
\hline Number of Nodes & 120 \\
\hline Movement model 1 & Random Waypoint \\
\hline Router & Epidemic \\
\hline Number of groups & 03 \\
\hline Buffer size & $2 \mathrm{MB}$ \\
\hline Transmission range & $10 \mathrm{M}$ \\
\hline Transmission speed & $250 \mathrm{~K}$ \\
\hline Message creation & $30-40$ (seconds) \\
\hline Simulations times & $200 \mathrm{k}$ \\
\hline Area & $4500 \mathrm{~m} \times 3400 \mathrm{~m}--8500 \mathrm{~m} \mathrm{x} \mathrm{7400m}$ \\
\hline TTL & Infinity \\
\hline
\end{tabular}

In the Second scenario we compare the results of FIFO and TMHF by varying the simulation area from $4500 \times 3400$ to 8500 $\mathrm{x} 7400$ with the increment of $1000 \mathrm{~m}$.

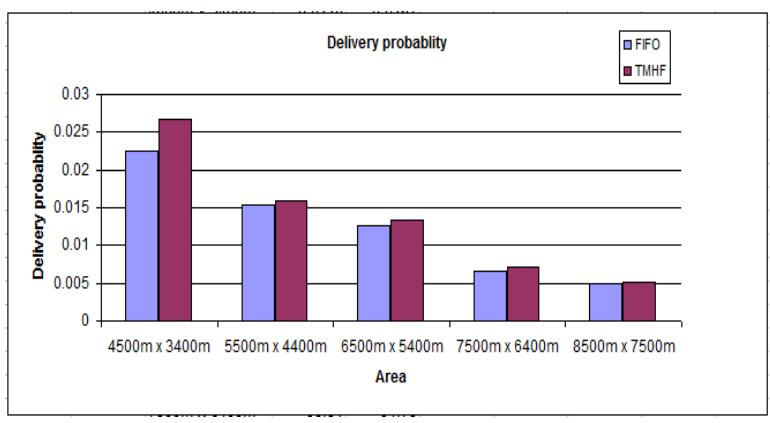

Fig 09 Delivery probability w.r.t Simulation area

From fig 9 shows the comparison of FIFO and TMHF queue modes in terms of delivery probability. We can analyze the results of TMHF proves better delivery probability as the area of simulation wider in terms of meters and nodes goes sparse with the PWP mobility models.

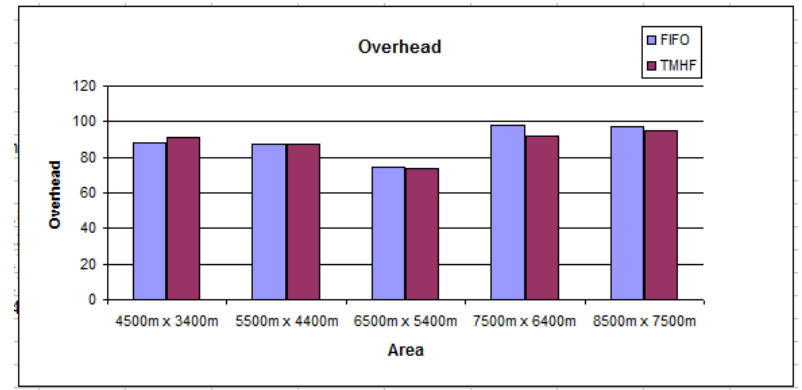

Fig 10 Overhead w.r.t Simulation area

Fig 10 measure the overhead of FIFO and TMHF strategies.

We can study that overhead gradually goes down by increasing the simulation area. 


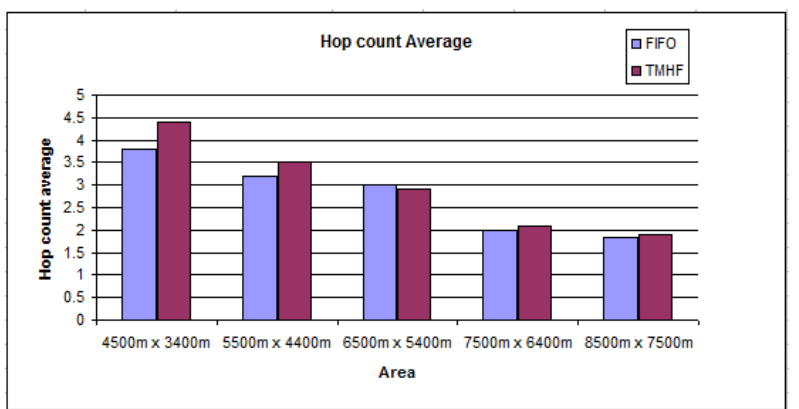

Fig 11 Hop count Average w.r.t Simulation area

Fig 11 demonstrates the impact of simulation area on hop count. As expected that the hop count in proposed TMHF is higher then FIFO.

\subsection{By Varying Transmission range}

Table 5. Simulation Time by varying time interval

\begin{tabular}{|c|c|}
\hline Number of Nodes & 120 \\
\hline Movement model 1 & Random Waypoint \\
\hline Router & Epidemic \\
\hline Number of groups & 03 \\
\hline Buffer size & $2 \mathrm{MB}$ \\
\hline Transmission range & $10 \mathrm{M}--120 \mathrm{M}$ \\
\hline Transmission speed & $250 \mathrm{~K}$ \\
\hline Message creation interval & 30-40 (seconds) \\
\hline Simulations times varies & $160 \mathrm{k}$ \\
\hline
\end{tabular}

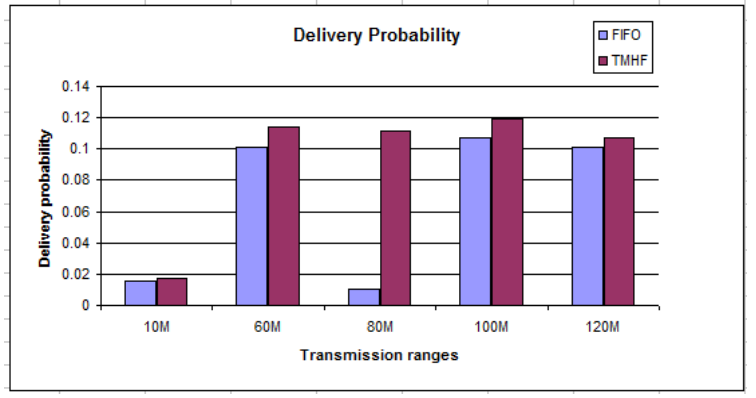

Fig 12 Delivery probability w.r.t Transmission range

Fig 12 determines the statistics of THMF and FIFO in terms of delivery probability. We can see that by increasing the transmission range THMF proves better delivery probability then FIFO. As the transmission range increases the nodes frequently encounters each other, hence by forwarding greater hop message prove high delivery probability.

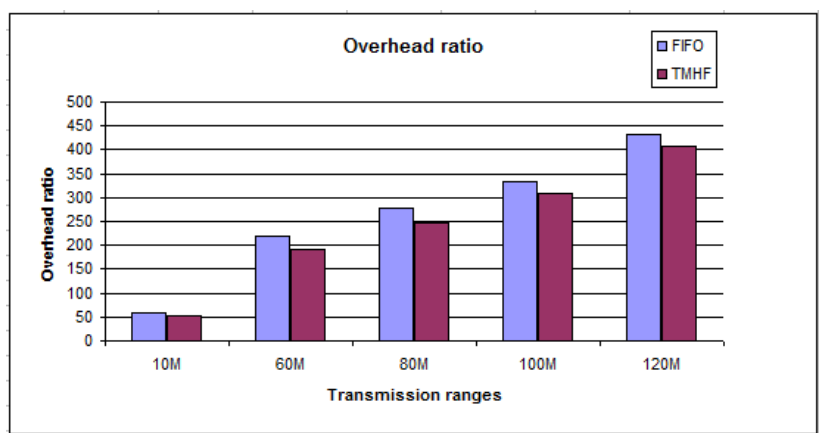

Fig 13 Overhead w.r.t Transmission range

In fig 13 we manipulate the results of overhead with FIFO and proposed TMHF strategies. Fig 13 we can see that at all transmission ranges the overhead of THMF is lower.

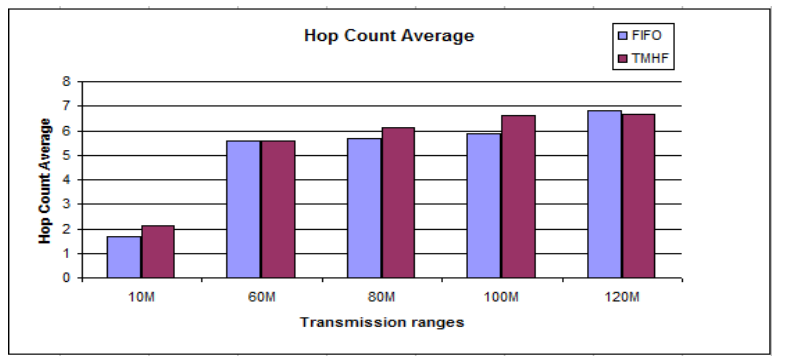

Fig 14 hop count Average w.r.t Transmission range

Fig 14 shows that TMHF have greater value of Hop count as compared to FIFO.

\section{CONCLUSION}

In this paper, we propose a forwarding strategy called as TMHF that optimizes the DTN epidemic routing protocol. The proposed policy reduce overhead and raise the delivery probability as compared to FIFO. The aim was to effectively utilize the network resources by giving priority to messages having higher value of hop count.

In order to check the accuracy of TMHF several experiments were performed at various time intervals by varying node transmission range and simulation area.

\section{REFERENCES}

[1] A.indgren and K. S. Phanse, "Evaluation of queuing policies and forwarding strategies for routing in intermittently connected networks,"in Proc. of IEEE COMSWARE, pp. 1-10, Jan. 2006.

[2] Qaisar Ayub, Sulma Rashid and Dr.Mohd Soperi Mohd Zahid. Article: Optimization of Epidemic router by new forwarding queue mode TSMF. International Journal of Computer Applications 7(11):5-8, October 2010. Published By Foundation of Computer Science.

[3] Lindgren, A. Doria, and O. Schelen, "Probabilistic routing in intermittently connected networks," SIGMOBILE Mobile Computing and Communication Review, vol. 7, no. 3, 2003.pp 19-20.

[4] KERÄNEN, A., AND OTT, J. Increasing Reality for DTN Protocol Simulations. Tech. rep., Helsinki University of Technology, Networking Laboratory, July 2007. 
[5] T. Spyropoulos, K. Psounis, and C. Raghavendra A, C. S. "Single-copy routing in intermittently connected mobile networks," IEEE/ACM Transactions on Networking (TON), vol. 16, pp. 63-76, Feb. 2008.

[6] Tara Small and Zygmunt Haas." The shared wireless infestation model - a new ad hoc networking paradigm (or where there is a whale, there is a way)", In Proceedings of The Fourth ACM International Symposium on Mobile Ad Hoc Networking and Computing (MobiHoc 2003), pages 233.244, June 2003.

[7] Philo Juang, Hidekazu Oki, Yong Wang, Margaret Martonosi, Li-Shiuan Peh, and Daniel Rubenstein. Energyef_cient computing for wildlife tracking: Design tradeoffs and early experiences with zebranet. In Proceedings of Tenth International Conference on Architectural Support for Programming Languages and Operating Systems (ASPLOS-X), San Jose, CA, October 2002.

[8] Vahdat and D. Becker. Epidemic Routing for Partiallyconnected Ad hoc Networks. Technical Report CS-200006, Duke University, July 2000.

[9] SCOTT, J., HUI, P., CROWCROFT, J., AND DIOT, C. Haggle: Networking Architecture Designed Around Mobile Users. In Proceedings of IFIP WONS (2006).

[10] FALL, K. A Delay-Tolerant Network Architecture for Challenged Internets. In Proc. of ACM SIGCOMM (2003).

[11] Z. J. Haas and T. Small. A New Networking Model for Biological Applications of Ad Hoc Sensor Networks. IEEE/ACM Transactions on Networking, 14, No. 1:27-40, 2006.

[12] T. Spyropoulos, K. Psounis, and C. Raghavendra. Efficient Routing in Intermittently Connected Mobile Networks: The Multi-copy Case. In ACM/IEEE Transactions on Networking, 2007.

[13] T. Small and Z. J. Haas. Resource and performance tradeoffs in delay-tolerant wireless networks. In SIGCOMM Workshop on Delay Tolerant Networking (WDTN), 2005.

[14] T. Spyropoulos, K. Psounis, and C. S. Raghavendra." Spray and wait: an efficient routing scheme for intermittently connected mobile networks", In SIGCOMM Workshop on Delay Tolerant Networking (WDTN), 2005

[15] Sulma Rashid, Qaisar Ayub, M. Soperi Mohd Zahid , A.Hanan. Abdullah. "Optimization of DTN routing protocols by using forwarding strategy (TSMF) and queuing drop policy (DLA)", International Journal of Computer and Network Security, Vol 2,ISSUE 10,OCTOBER 2010. pp .71-75.

[16] P. Zhang, C. M. Sadler, S. A. Lyon, and M. Martonosi. Hardware Design Experiences in ZebraNet. In Proc. ACM SenSys, pages 227-238, Nov. 2004.

[17] Maffei, K. Fall, and D. Chayes. Ocean Instrument Internet. In Proc. AGU Ocean Sciences Conf., Feb 2006.
[18] J. Partan, J. Kurose, and B. N. Levine. A Survey of Practical Issues in Underwater Networks. In Proc. ACMWUWNet, pages 17-24, Sept. 2006.

[19] J. Ott and D. Kutscher. A Disconnection-Tolerant Transport for Drive-thru Internet Environments. In Proc. IEEE INFOCOM, pages 1849-1862, Mar. 2005.

[20] J. Burgess, B. Gallagher, D. Jensen, and B. N. Levine. MaxProp: Routing for Vehicle-Based Disruption- Tolerant Networks. In Proc. IEEE Infocom, April 2006.

[21] Sulma Rashid,Qaisar Ayub,"Effective buffer management policy DLA for DTN routing Protocals under congetion", International Journal of Computer and Network Security, Vol 2,NO 9,Sep 2010. pp .118-121

[22] Rossi, M., M. Zorzi, and R.R. Rao, Statistically assisted routing algorithms (SARA) for hop count based forwarding in wireless sensor networks. Wireless Networks, 2008. 14(1): p. 55-70.

[23] Sulma Rashid, Qaisar Ayub, Soperi Mohd M Zahid and A.Hanan. Abdullah. Article: E-DROP: An Effective Drop Buffer Management Policy for DTN Routing Protocols. International Journal of Computer Applications 13(7):813, January 2011. Published by Foundation of Computer Science

[24] Qaisar Ayub, Sulma Rashid," T-Drop: An optimal buffer management policy to improve QOS in DTN routing protocols", Journal of Computing, Vol 2, ISSUE 10, OCTOBER 2010. pp .46-50.

\section{AUTHORS PROFILE}

Sulma Rashid She has received her MS Degree in computer science in 2007 from IQRA University Islamabad Pakistan and MCS degree in 2001 from UAAR Pakistan. She has 10 years of teaching experience. Her areas of interest are DTN, Adhoc, security, Network programming, Operating system, wireless networks and MANETS. She is currently PHD Scholar at UTM.

Qaisar Ayub He has obtained his MCS Computer Science degree in 2005 from Comsat Institute of Information Technology Pakistan, and BCS (Hons.) computer science from Allama Iqbal Open University Pakistan in 2003. He has 5 years of experience in conducting professional trainings (Oracle, Java, ) and software development

M. Soperi Mohd Zahid He received Ph.D degree in Computer Science from University of Wisconsin, Milwaukee in 2009, M.S. degree in Computer Integrated Manufacturing from Rochester Institute of Technology, New York and B.S. degree in 1988 from New Mexico State University, Las Cruces in Computer Science and Mathematics .He is currently a faculty member in Computer Science, Universiti Teknologi Malaysia. His research interests include Internet Routing Protocols and Delay/Disruption Tolerant Networks. 